\title{
Comparison of Comorbid Conditions Between Cancer Survivors and Age-Matched Patients Without Cancer
}

\author{
Satyajeet Roy ${ }^{\mathrm{a}, \mathrm{d}}$, Shirisha Vallepu ${ }^{\mathrm{b}}$, Cristian Barrios ${ }^{\mathrm{a}}$, Krystal Hunter ${ }^{\mathrm{c}}$
}

\begin{abstract}
Background: Cancer survivors suffer from many comorbid conditions even after the cure of their cancers beyond 5 years. We explored the differences in the association of comorbid conditions between the cancer survivors and patients without cancer.
\end{abstract}

Methods: Electronic medical records of 280 adult cancer survivors and 280 age-matched patients without cancer in our suburban internal medicine office were reviewed.

Results: Mean age of the cancer survivors was $72.5 \pm 13.1$ years, and the age of the patients without cancer was $72.5 \pm 12.8$ years. The number of male cancer survivors was significantly higher than the female cancer survivors $(52.5 \%$ vs. $47.5 \%, \mathrm{P}<0.001)$. There were significantly more Caucasians and other races (majority Asians) in the cancer survivor group compared to the patients without cancer group ( $81.8 \%$ vs. $79.3 \%$ and $4.6 \%$ vs. $0.4 \%$, respectively, $\mathrm{P}<0.05)$; while there were significantly less African Americans and Hispanics in the cancer survivor group compared to the patients without cancer group ( $10.0 \%$ vs. $12.8 \%$ and $3.6 \%$ vs. $7.5 \%$, respectively, $\mathrm{P}<0.05)$. Hypertension $(64.3 \%)$, hyperlipidemia $(56.1 \%)$, osteoarthritis $(34.3 \%)$, hypothyroidism $(21.8 \%)$, diabetes mellitus $(21.8 \%)$ and coronary artery disease $(21.8 \%)$ were the most common comorbid conditions observed in the cancer survivors. Osteoarthritis was the only comorbid condition that was significantly less frequently associated with the cancer survivors compared to the patients without cancer $(42.9 \%$, $\mathrm{P}<0.05)$. The frequencies of all other comorbid conditions were not significantly different between the two groups. The majority of our group of cancer survivors had one or more types of the top six cancers which include prostate cancer $(30.7 \%)$, melanoma (13.9\%), thyroid cancer (11.4\%), colon cancer (11.1\%), uterine cancer $(11.1 \%)$ and urinary bladder cancer $(11.1 \%)$; while only a few had cancer of the

Manuscript submitted September 9, 2018, accepted October 8, 2018

aDepartment of Medicine, Cooper University Hospital, Cooper Medical School of Rowan University, Camden, NJ, USA

bSoutheast University, Nanjing, China

'Cooper Research Institute, Cooper Medical School of Rowan University, Camden, NJ, USA

${ }^{\mathrm{d} C}$ Corresponding Author: Satyajeet Roy, Department of Medicine, Cooper University Hospital, Cooper Medical School of Rowan University, 1103 North Kings Highway, Suite 203, Cherry Hill, NJ 08034, USA.

Email: roy-satyajeet@cooperhealth.edu

doi: https://doi.org/10.14740/jocmr3617w cervix $(6.1 \%)$ or breast cancer $(0.3 \%)$. Use of aspirin, statin, vitamin $\mathrm{D}$, multivitamins, metformin and fish oil supplement in the cancer survivors was similar to the patients without cancer.

Conclusions: Hypertension, hyperlipidemia, osteoarthritis, hypothyroidism, diabetes mellitus and coronary artery disease are the most common associated comorbid conditions in the cancer survivors. Osteoarthritis is less frequently seen in the cancer survivors compared to the patients without cancer. The frequencies of other comorbid conditions are not significantly different between the two groups.

Keywords: Cancer survivors; Comorbid medical conditions; Comorbidities in cancer survivors

\section{Introduction}

Cancer is the second most leading cause of death in the United States. About $22 \%$ of deaths in 2015 were caused by cancer [1]. In 2018 there were 1,735,350 new cancer cases in the United States [1]. The four most common cancers in the US are breast cancer, prostate cancer, lung cancer and colorectal cancer, which accounted for $46 \%$ of total cancer deaths in 2015. From 2006 to 2015 the cancer death rate has decreased about $1.5 \%$ annually in both men and women, whereby accounting for more cancer survivors every year [1].

There are several definitions of cancer survivorship. The National Cancer Institute defines a cancer survivor as an individual diagnosed with cancer, starting from the time of diagnosis through the balance of their life [2]. Deimling and colleagues defined cancer survivors as older adults who have survived cancers for at least 5 years [3]. The office of cancer survivorship at the National Cancer Institute defines cancer survivorship as survivors of cancer 5 years after the diagnosis [4]. Even though there is a widespread accepted definition of 5 -year cutoff, it is not appropriate for some types of cancers, such as lung cancer and pancreatic cancer where the majority of patients die within 1 year of diagnosis $[5,6]$. Cancer survivorship differs widely based on the organ system involved. For example, the 5-year survival rate of prostate cancer is $99 \%$, melanoma is $92 \%$, breast cancer is $90 \%$, urinary bladder cancer is $77 \%$, kidney is $74 \%$, non-Hodgkin lymphoma is $71 \%$, uterine and cervix cancer is $67 \%$, colorectal cancer is $65 \%$, cancer of oral cavity and pharynx is $65 \%$, ovarian cancer is $47 \%$, esophageal cancer is $19 \%$, liver cancer is $18 \%$, lung can- 
cer is $18 \%$ and pancreatic cancer is $8 \%$ [1]. In any case, the cancer survivors not only have their primary cancer diagnosis to manage, but they also have other medical comorbidities that influence their health.

As the cancer survivors live longer, the prevalence of comorbid conditions tend to increase [7]. An estimated prevalence of comorbidities is highest in patients with lung cancer $(58 \%)$, kidney cancer $(54 \%)$, stomach cancer $(53 \%)$, bladder cancer $(53 \%)$ and prostate cancer $(51 \%)$ [8]. Comorbidities and their influence on patient's quality of life also depend on the site of the cancer. For example, hypertension and diabetes specifically were associated with poorer quality of life in breast cancer survivors [9], while the most common concomitant illnesses in patients with colorectal cancer were cardiovascular disease, hypertension, and previous cancers [10]. Several studies have shown that patients with comorbidities have higher mortality than patients without comorbidities [11]. A study by Koroukian and colleagues showed that the proportion of cancer patients with no comorbidities, disabilities, or geriatric syndromes decreased gradually with increasing age [12]. This increase in comorbidities can have significant and wide-ranging implications for cancer patients. These implications include: long-term survival [13-16], staging of a patients' cancers at diagnosis $[16,17]$, and treatment options [18]. Therefore, it becomes imperative to gather more information regarding comorbid conditions in the cancer survivors. We investigated the comorbid conditions associated with the cancer survivors. We hypothesized that the types of comorbidities differ between cancer survivors and patients who do not have cancer.

\section{Materials and Methods}

\section{Study selection}

This study was a retrospective electronic medical record review that observed the comorbidities between cancer survivors and age-matched patients without cancer. Patients were seen in a single suburban primary care office between January 1, 2017 and December 31, 2017. The inclusion criteria was adult patients of age 18 years or older who had a cancer diagnosis made at least 5 years prior to the time of their most recent clinical visit as per the documentation in the electronic medical record. An age-matched group of patients who did not have a cancer diagnosis were also included for comparison. The exclusion criterion was patients under the age of 18 years and patients who died due to cancer within 5 years of diagnosis. The study was reviewed and approved by the Institutional Review Board of the Cooper Health System, Camden, New Jersey, USA.

\section{Data collection}

The following data were collected for each patient: age, gender, race (Caucasian, African American, Hispanic or other), social history (tobacco use, alcohol use, and/or recreational

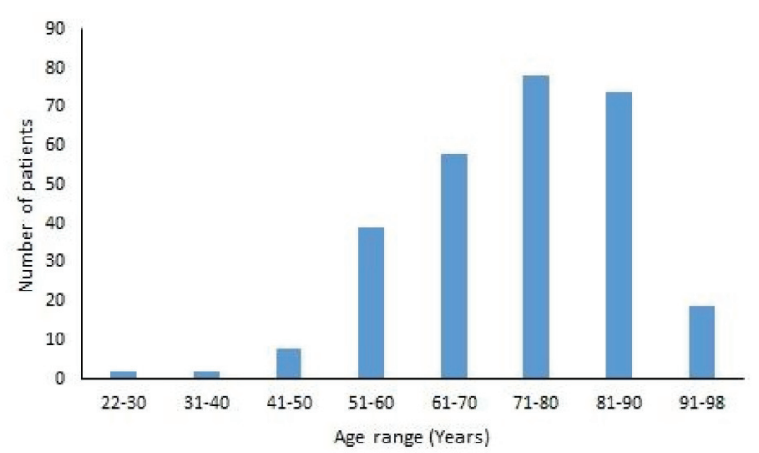

Figure 1. Age distribution of the cancer survivors.

drug use), comorbid medical conditions, such as hypertension, hyperlipidemia, hypothyroidism, coronary artery disease, cerebral vascular attack, carotid artery stenosis, peripheral arterial disease, congestive heart failure, chronic obstructive pulmonary disease, asthma, chronic kidney disease, liver disease, diabetes, hypothyroidism, other endocrine disorders, psychiatric conditions (depression, bipolar, anxiety disorder, schizophrenia), osteoarthritis and other rheumatologic diseases, vitamin $\mathrm{D}$ deficiency, presence or absence of cancer and the type of cancer. We also collected the data on use of some over-thecounter or prescription medications, such as aspirin, statin, vitamin D, multivitamin, metformin and fish oil.

\section{Statistical study}

Collected data were entered into a Microsoft Excel (2016, Redmond, Washington, USA) spreadsheet. Statistical analysis was done using SPSS (Statistical Package for the Social Sciences, version 15.01, IBM, Armonk, New York, USA). Subjects were divided into two groups. Group 1 represented the patients who were cancer survivors. Group 2 represented the age-matched patients without cancer. Chi-square test was used to compare the categorical variables. Independent $t$-test and Mann Whitney U test were used to compare the continuous variables between the groups. Independent $t$-test was used to compare the means of normally distributed variables while Mann Whitney U test was used to compare ranks of nonnormally distributed variables. In this study, significance was defined as a $\mathrm{P}<0.05$.

\section{Results}

A total of 560 patient medical records were included. Among them, $280(50 \%)$ patients were cancer survivors (Group 1) and $280(50 \%)$ patients were age-matched patients who had no history of cancer (Group 2). In the cancer survivor group 51 $(18.2 \%)$ patients had more than one types of cancers.

The age range of the cancer survivors in our study was 22 to 98 years. The majority of the cancer survivors were in the 61 to 90 years age range (Fig. 1). The mean age of the patients in the cancer survivor group was $72.5 \pm 13.1$ years and in the patients without cancer group was $72.5 \pm 12.8$ years. There 
Table 1. Baseline Characteristics

\begin{tabular}{|c|c|c|c|}
\hline Variable & Group 1 (cancer survivors) $(n=280)$ & Group 2 (patients without cancer) $(n=280)$ & $\mathbf{P}$ \\
\hline Age mean (SD) & $72.5(13.1)$ & $72.5(12.8)$ & $<0.938^{*}$ \\
\hline \multicolumn{4}{|l|}{ Gender } \\
\hline Female (n, \%) & $133(47.5)$ & $199(71.1)$ & $<0.001^{\#}$ \\
\hline Race & & & $<0.001^{\#}$ \\
\hline Hispanic (n, \%) & $10(3.6)$ & $21(7.5)$ & $<0.05^{\#}$ \\
\hline Other $(\mathrm{n}, \%)$ & $13(4.6)$ & $1(0.4)$ & $<0.05^{\#}$ \\
\hline \multicolumn{4}{|l|}{ Social factors } \\
\hline Alcohol (n, \%) & $158(56.4)$ & $152(54.2)$ & $0.651^{\#}$ \\
\hline
\end{tabular}

*Paired $t$-test. \#McNemar test.

was no statistically significant difference in the mean ages between the two groups (Table 1). Gender analysis revealed that the number of male cancer survivors was significantly higher than the female cancer survivors $(52.5 \%$ vs. $47.5 \%, \mathrm{P}<0.001)$. Intra-group comparison showed that there were significantly less female patients (47.5\%) who were cancer survivors compared to the female patients without cancer $(71.1 \%, \mathrm{P}<0.001)$. There were significantly more male cancer survivors in our mean age group of patients $(52.5 \%)$ compared to the patients without cancer $(28.9 \%, \mathrm{P}<0.001)$ (Table 1$)$.

The analysis of race showed that the majority of the patients in the cancer survivor group were Caucasians $(81.8 \%)$, followed by African Americans (10.0\%), other races (4.6\%) and Hispanics (3.6\%) (Table 1). A similar hierarchy of the frequency of races was observed in the patients without cancer group except for more Hispanics $(7.5 \%)$ compared to other races $(0.4 \%)$. There were significantly more Caucasians and other races (majority Asians) in the cancer survivor group compared to the patients without cancer group (81.8\% vs. $79.3 \%$ and $4.6 \%$ vs. $0.4 \%$, respectively; $\mathrm{P}<0.05$ ), while there were significantly less African Americans and Hispanics in the cancer survivor group compared to the patients without cancer group (10.0\% vs. $12.8 \%$ and $3.6 \%$ vs. $7.5 \%$, respectively; $\mathrm{P}<$ $0.05)$ (Table 1).

Social factor analysis, such as alcohol intake and cigarette smoking showed that about half of the patients in both the groups had a history of alcohol use and cigarette smoking, but there was no statistically significant difference between the two groups (Table 1).

Hypertension, hyperlipidemia, osteoarthritis, hypothyroidism, diabetes mellitus and coronary artery disease were the most common comorbid conditions observed in the cancer survivors (Table 2). We found that hypertension was an associated comorbid condition in about two-thirds of the cancer survivor group and in the patients without cancer group. There was no statistically significant difference between the two groups (Table 2). About half of the patients in both the groups had hyperlipidemia. The difference was not statistical- ly significant. On the other hand, osteoarthritis was a less associated comorbid condition in the cancer survivors compared to the patients without cancer $(34.3 \%$ vs. $42.9 \%)$. The difference was statistically significant $(\mathrm{P}<0.05)$ (Table 2$)$. About a fifth of the patients had hypothyroidism, diabetes mellitus and coronary artery disease in both the groups. There was no statistically significant difference between the two groups (Table 2, Fig. 2).

We found vitamin D deficiency, anxiety disorder, depression, asthma and chronic kidney disease were other comorbid conditions that were associated in about one-fifth to one-tenth of the cancer survivors. The frequency of association of these comorbid conditions was similar in the patients without cancer. The difference was not statistically significant (Table 2). Other less commonly associated comorbid conditions were chronic obstructive airway disease, cerebrovascular accident, peripheral arterial disease, congestive heart failure, carotid artery stenosis, bipolar disorder, liver disease and schizophrenia. The difference in the frequencies of these comorbid conditions was not statistically significant between the two groups (Table 2, Fig. 2).

In our study the top six cancers that were associated with the majority of the cancer survivors $(87.5 \%)$ were prostate $(30.7 \%)$, melanoma $(13.9 \%)$, thyroid $(11.4 \%)$, colon $(11.1 \%)$ uterus $(11.1 \%)$ and urinary bladder (9.3\%) (Table 3). Cancer of cervix, meningioma, essential thrombocythemia, pituitary tumors, gastroesophageal cancer, anorectal cancer and skin cancers other than melanoma were less frequently associated with the cancer survivors with the frequencies of $6.1 \%$, $5.3 \%, 3.9 \%, 3.9 \%, 2.1 \%, 1.8 \%$ and $1.4 \%$, respectively (Table 3, Fig. 3). As mentioned earlier, $18.2 \%$ of patients had more than one types of cancers. There were extremely low prevalence of other cancers in the cancer survivors, such as cancers of connective and soft tissues, esophagus, brain, larynx, and liver. The prevalence of cancers, such as acoustic neuroma, breast, endotheliosarcoma, ependymoma, esthesioneuroblastoma, myelodysplastic syndrome, cancer of parathyroid, parotid gland, pharynx, tonsil and tongue were even lower 
Table 2. Associated Comorbid Conditions

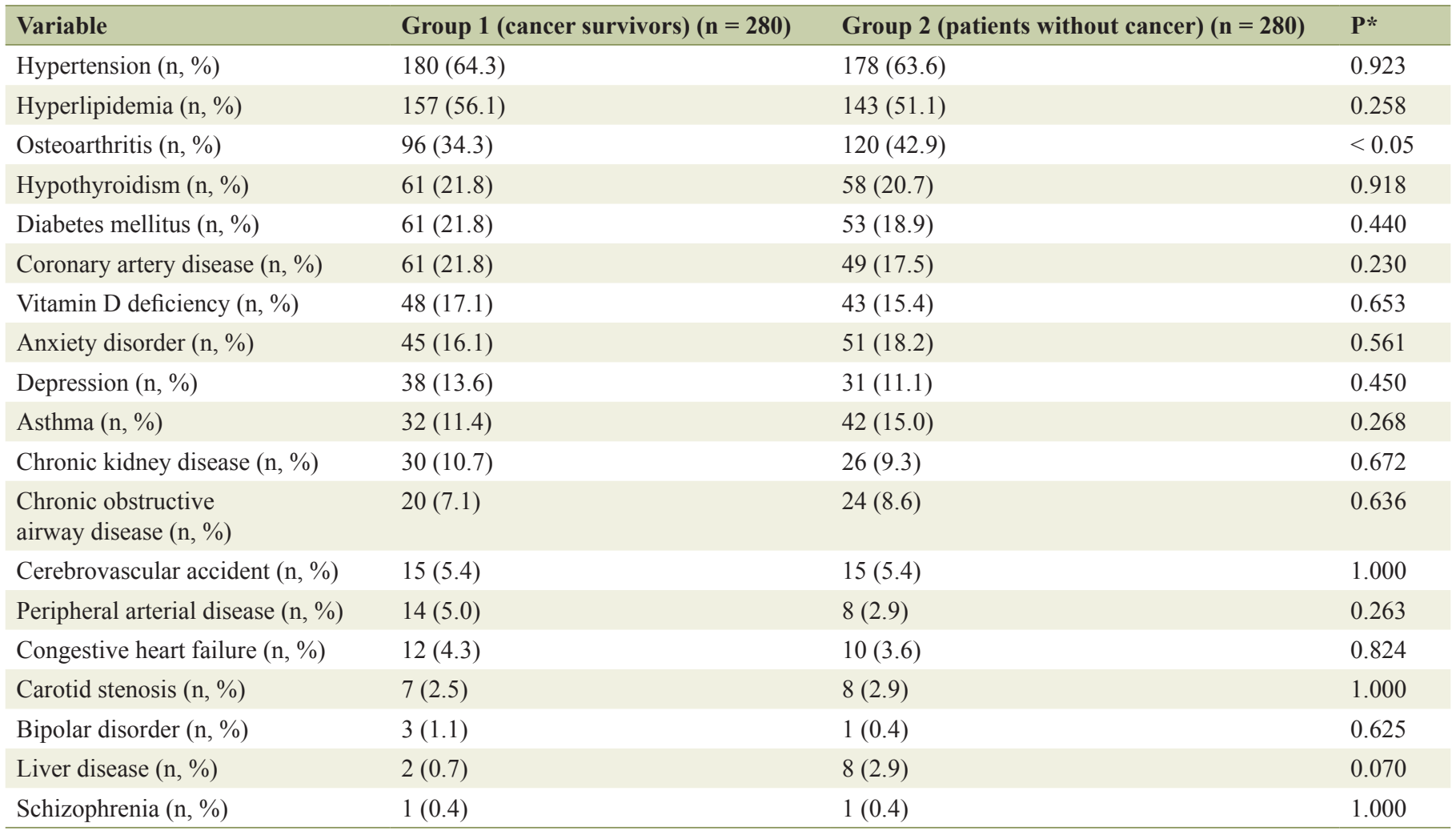

${ }^{*}$ McNemar test.

(Table 3).

A further analysis of the top six types of cancers in our cancer survivors (Fig. 4) showed that hypertension was the most common comorbid condition seen in the cancer survivors of prostate cancer $(77.9 \%)$, uterine cancer $(77.4 \%)$, urinary bladder cancer $(76.9 \%)$ and colon cancer (58.1) (Table 4$)$. In the cancer survivors of melanoma, hyperlipidemia was the most commonly associated comorbid medical condition (71.8\%). Hypothyroidism was the most common comorbid condition seen in the cancer survivors of thyroid cancer (65.6\%) (Table
4).

We found that about half of the patients in the cancer survivor group were taking aspirin and statin. The frequencies of aspirin and statin use were similar in the patients without cancer group. The difference was not statistically significant (Table 5). Similarly the use of vitamin D, multivitamin, metformin and fish oil was also observed in the cancer survivors with the frequencies of $36.1 \%, 20.7 \%, 13.2 \%$ and $5.0 \%$, respectively. The difference in the frequencies of use of the aforementioned over-the-counter or prescription medications was not statisti-

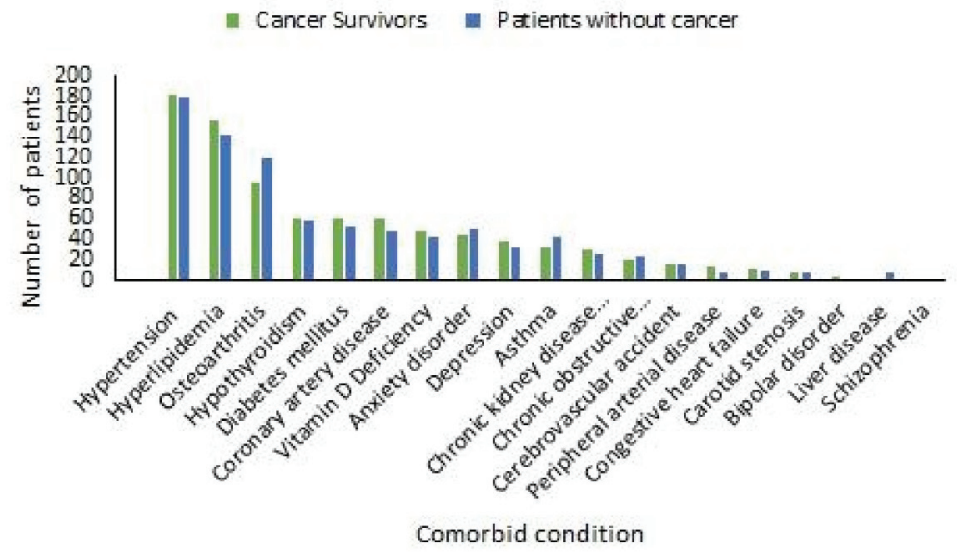

Figure 2. Frequencies of comorbid conditions in cancer survivors and patients without cancer. 
Table 3. Frequency of Types of Cancers in the Cancer Survivors

\begin{tabular}{ll}
\hline Type of cancer & Frequency $(\mathbf{n}=\mathbf{2 8 0})$ \\
\hline Prostate (n, \%) & $86(30.7)$ \\
Melanoma (n, \%) & $39(13.9)$ \\
Thyroid (n, \%) & $32(11.4)$ \\
Colon (n, \%) & $31(11.1)$ \\
Uterus (n, \%) & $31(11.1)$ \\
Urinary bladder (n, \%) & $26(9.3)$ \\
Cervix (n, \%) & $17(6.1)$ \\
\hline Meningioma (n, \%) & $15(5.3)$ \\
Essential thrombocythemia (n, \%) & $11(3.9)$ \\
Pituitary (n, \%) & $11(3.9)$ \\
Gastroesophageal (n, \%) & $6(2.1)$ \\
Ano-rectum (n, \%) & $5(1.8)$ \\
Skin-non melanoma (n, \%) & $4(1.4)$ \\
Connective and soft tissue (n, \%) & $3(1.1)$ \\
Esophagus (n, \%) & $3(1.1)$ \\
\hline Brain (n, \%) & $2(0.7)$ \\
Larynx (n, \%) & $2(0.7)$ \\
\hline Liver (n, \%) & $2(0.7)$ \\
Acoustic neuroma (n, \%) & $1(0.3)$ \\
Breast (n, \%) & $1(0.3)$ \\
Endotheliosarcoma (n, \%) & $1(0.3)$ \\
\hline Ependymoma (n, \%) & $1(0.3)$ \\
Esthesioneuroblastoma (n, \%) & $1(0.3)$ \\
Myelodysplastic syndrome (n, \%) & $1(0.3)$ \\
Parathyroid (n, \%) & $1(0.3)$ \\
Parotid (n, \%) & $1(0.3)$ \\
Pharynx (n, \%) & $1(0.3)$ \\
Tonsil (n, \%) & \\
Tongue (n, \%) & \\
\hline
\end{tabular}

cally significant between the two groups.

\section{Discussion}

We found two major findings. First, hypertension, hyperlipidemia, osteoarthritis, hypothyroidism, diabetes mellitus and coronary artery disease were the most common comorbid conditions observed in the cancer survivors. Osteoarthritis was the only comorbid condition that was significantly less frequently associated with the cancer survivors compared to the patients without cancer, while the frequencies of association of all other comorbid conditions were not different between the groups.

Second, our group of cancer survivors had a history of one or more types of the top six cancers, which include prostate cancer, melanoma, thyroid cancer, colon cancer, uterine cancer and urinary bladder cancer. History of cancer of cervix, gastroesophageal cancer, anorectal cancer and skin cancers other than melanoma was less frequently seen in our group of cancer survivors. History of breast cancer was extremely rare in our group of cancer survivors.

Many studies have shown that hypertension is the most common comorbid condition seen in the cancer survivors. The prevalence of hypertension in the cancer survivors ranges from $20 \%$ to $65 \%$ [7, 19-24]. In our study hypertension was the leading comorbid condition observed in the cancer survivors with a prevalence of $64.3 \%$. Although some studies have found a higher prevalence of hypertension in the cancer survivors compared to patients with no history of cancer based on either certain cancer types or specific treatment methods [20,24, 25], we found no significant difference in the prevalence of hypertension between cancer survivors and age-matched group of patients without cancer. Our findings were similar to the findings published by Enright and colleagues [26]. We also found that hypertension was the most common associated comorbid condition in our group of cancer survivors of prostate cancer, uterine cancer, urinary bladder cancer and colon cancer.

A study by Guy and colleagues found $54.8 \%$ prevalence of hyperlipidemia in the cancer survivors which was statistically significantly higher than the prevalence in the patients without history of cancer (28.7\%) [27]. In our study, we found a similarly high prevalence of hyperlipidemia in the cancer survivors $(56.1 \%)$ which was higher than our group of agematched patients without cancer (51.1\%), but the difference was not statistically significant. Our findings were similar to the findings of Enright and associates who found a $54.7 \%$ prevalence of hyperlipidemia in the cancer survivors which was not statistically different than the patients without cancer [26]. Among the top six types of the cancer survivors in our study, the survivors of melanoma had the highest prevalence of hyperlipidemia (71.8\%) followed by the survivors of bladder cancer, uterine cancer, prostate cancer, colon cancer and thyroid cancer. The association of high prevalence of hyperlipidemia in cancer survivors of melanoma was a unique finding in our study since we did not find any study that had looked into the prevalence of hyperlipidemia in the cancer survivors who had melanoma.

In our study, the third most common comorbid condition observed in the cancer survivors was osteoarthritis. Although osteoarthritis was seen in about one-third of the cancer survivors, its prevalence was significantly less in the cancer survivors when compared to the patients without cancer. We did not find any published study documenting the prevalence of osteoarthritis in the cancer survivors. Hence, we believe that our study is the first study reporting a significantly lower prevalence of osteoarthritis in the cancer survivors compared to the age-matched patients without cancer. The cancer survivors of colon cancer had the lowest prevalence of osteoarthritis followed by prostate cancer survivors and uterine cancer survivors. Further studies are needed to find out the etiological factors influencing the significant difference in the lower association of osteoarthritis in the cancer survivors compared to the patients without cancer.

Hypothyroidism is a known comorbid condition in certain 


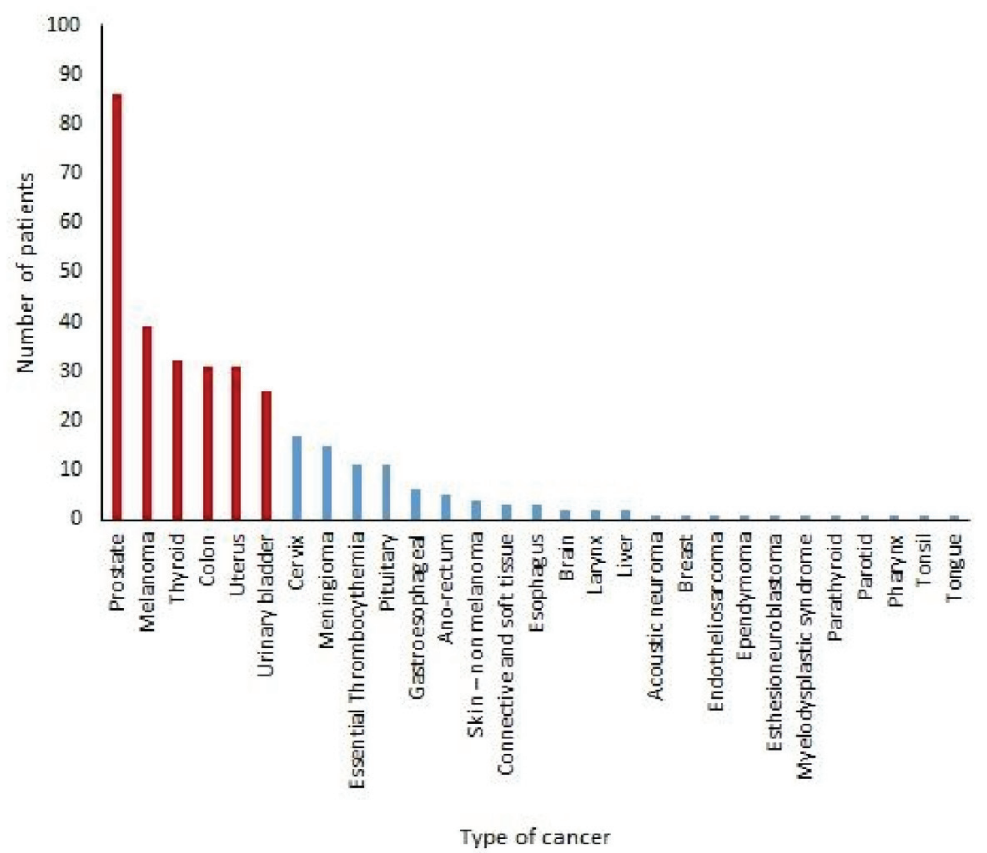

Figure 3. Frequencies of types of cancers in cancer survivors.

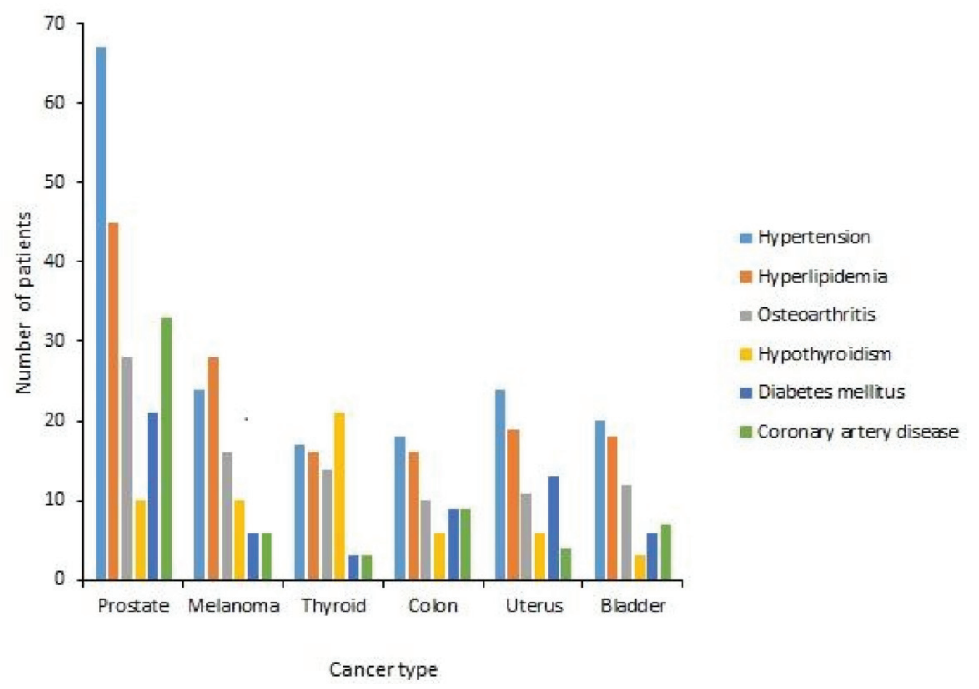

Figure 4. Frequencies of comorbid conditions in cancer survivors of top six types of cancers.

Table 4. Associated Comorbid Conditions in Specific Cancer Survivors Based on Cancer Types

\begin{tabular}{|c|c|c|c|c|c|c|}
\hline Comorbid condition & $\begin{array}{l}\text { Prostate } \\
(n=86)\end{array}$ & $\begin{array}{l}\text { Melanoma } \\
(\mathrm{n}=39)\end{array}$ & $\begin{array}{l}\text { Thyroid } \\
(n=32)\end{array}$ & $\begin{array}{l}\text { Colon } \\
(n=31)\end{array}$ & $\begin{array}{l}\text { Uterus } \\
(n=31)\end{array}$ & $\begin{array}{l}\text { Bladder } \\
(n=26)\end{array}$ \\
\hline Hypertension (n, \%) & $67(77.9)$ & $24(61.5)$ & $17(53.1)$ & $18(58.1)$ & $24(77.4)$ & $20(76.9)$ \\
\hline Osteoarthritis (n, \%) & $28(32.6)$ & $16(41.0)$ & $14(43.8)$ & $10(32.3)$ & $11(35.5)$ & $12(46.1)$ \\
\hline Hypothyroidism (n, \%) & $10(11.6)$ & $10(25.6)$ & $21(65.6)$ & $6(19.3)$ & $6(19.4)$ & $3(11.5)$ \\
\hline
\end{tabular}


Table 5. Commonly Used Drugs and Supplements

\begin{tabular}{llll}
\hline Variable & Group 1 (cancer survivors) $(\mathbf{n}=\mathbf{2 8 0})$ & Group 2 (patients without cancer) $(\mathbf{n}=\mathbf{2 8 0})$ & P* \\
\hline Aspirin (n, \%) & $156(55.7)$ & $124(44.3)$ & 0.403 \\
Statin (n, \%) & $144(51.4)$ & $138(49.3)$ & 0.668 \\
Vitamin D (n, \%) & $101(36.1)$ & $103(36.8)$ & 0.923 \\
Multivitamin (n, \%) & $58(20.7)$ & $46(16.4)$ \\
Metformin (n, \%) & $37(13.2)$ & $32(11.4)$ & 0.224 \\
Fish Oil (n, \%) & $14(5.0)$ & $10(3.6)$ & 0.603 \\
\hline
\end{tabular}

${ }^{*} \mathrm{McNemar}$ test.

specific types of cancer survivors, such as cancers of head and neck. The Head and Neck Cancer Survivorship Care Guidelines from the American Society of Clinical Oncology recommends the primary care physicians to monitor thyroid-stimulating hormone levels every 6 - 12 monthly for the survivors of head and neck cancers [28]. In our study, we found that about one-fifth of our group of cancer survivors had hypothyroidism which was comparable to our age-matched (mean age 72.5 year) group of patients without cancer. Although the prevalence of hypothyroidism is about $6 \%$ in the eighth decade of life [29], our age-matched patients without cancer had a much higher prevalence compared to the trend in the general population of the United States. The prevalence of hypothyroidism in our group of cancer survivors was much higher than the findings of Chin and associates, who found that after a 7.5-year follow-up, $8 \%$ of their cancer survivors were diagnosed with hypothyroidism [30]. We also found that about two-thirds of the cancer survivors with history of thyroid cancer had hypothyroidism which is not unusual depending upon the modality of the treatment, such as total versus partial thyroidectomy, or radiation-based treatment of the thyroid gland.

We found that about one-fifth of our group of cancer survivors had diabetes mellitus. Various studies have reported $8 \%$ to $32 \%$ prevalence of diabetes in the cancer survivors [24, 3135]. Our findings fall in the similar range. Lega and colleagues found a $55 \%$ higher rate of diabetes among the childhood cancer survivors after a mean follow-up of 11.2 years [36]. They also found that the survivors of leukemia and lymphoma had the greatest risk of developing diabetes. Although more studies have found higher prevalence rates of diabetes in the cancer survivors compared to patients without cancer [24, 33], we found no significant difference in the prevalence of diabetes between the cancer survivors and the age-matched group of patients without cancer. Among the top six types of the cancer survivors in our study, the survivors of uterine cancer had the highest prevalence of diabetes $(42.9 \%)$ followed by the survivors of colorectal cancer, prostate cancer, bladder cancer, melanoma and thyroid cancer. The association of high prevalence of diabetes in the uterine cancer survivors was a unique finding in our study since we did not find any study that has reported the prevalence of diabetes in the survivors of uterine cancer.

There are many studies that have shown a higher prevalence of coronary artery disease and congestive heart failure in the cancer survivors [37-45]. The increased cardiovascular risk in the cancer survivors have been attributed secondary to a specific treatment for their cancers, such as hematopoietic stem cell transplantation [37], radiation or anthracycline therapy [38-41], use of trastuzumab, sorafenib and sunitinib, bevacizumab and other agents [42-44], and other cardiotoxicities [45]. Additionally, suboptimal control of multiple cardiovascular risk factors, such as hypertension, diabetes and hyperlipidemia, was also observed in the cancer survivors [26]. Mertens and colleagues found a 7-fold increase in mortality in the survivors of childhood cancers due to cardiovascular disease when compared to the age-matched population [46]. Although we found a higher prevalence of coronary artery disease in our cancer survivors $(21.8 \%)$ compared to the agematched patients without cancer $(17.5 \%)$, but the difference was not statistically significant in our study.

We found one or more types of the top six cancers in our group of cancer survivors, which include prostate cancer, melanoma, thyroid cancer, colon cancer, uterine cancer and urinary bladder cancer. Our findings match with the observations of Miller and colleagues who found prostate cancer, colorectal cancer and melanoma were the top three types of cancers in the male cancer survivors, but differ from their findings in the female cancer survivors in whom history of breast cancer was the predominant cancer type, followed by uterine cancer and colorectal cancer [47]. We found that only $0.3 \%$ of cancer survivors in our group had history of breast cancer. Although the 5 -year survival rates for breast cancer survivors range from $22 \%$ to $100 \%$ [48] depending upon the stage (e.g., IV to 0 , respectively), our findings are most likely due to less representation of breast cancer survivors in our sample of patient population.

In our study, we found significantly more male cancer survivors than female cancer survivors. Our findings differ from the 5-year cancer survival data from the Surveillance, Epidemiology and End Results (SEER) Database by sex from 1977 to 2006 which showed lower rates of survival for males than females [49].

The major strength of our study was a large sample size of cancer survivors and age-matched patient population without cancer from one office location. Subsequent follow-up patient visits with the same small group of healthcare providers allowed proper documentation of comorbid conditions in the electronic medical record. Nevertheless, our study had a few limitations, such as retrospective analysis allowed collection of only documented variables, and patient selection limited to a suburban outpatient population which limits generalization. 
We conclude that hypertension, hyperlipidemia, osteoarthritis, hypothyroidism, diabetes mellitus and coronary artery disease are the most commonly associated comorbid conditions in the cancer survivors. Osteoarthritis is less frequently seen in the cancer survivors compared to the patients without cancer. Excluding osteoarthritis, the frequencies of comorbid conditions are not significantly different between cancer survivors and age-matched patients without cancer.

\section{Acknowledgments}

The authors thank Christine Rickette RN (study coordinator) for her contribution to this study.

\section{Grant Support}

None.

\section{Conflict of Interest}

None.

\section{References}

1. Siegel RL, Miller KD, Jemal A. Cancer statistics, 2018. CA Cancer J Clin. 2018;68(1):7-30.

2. Division of Cancer Control and Population Sciences, National Cancer Institute, National Institutes of Health. Office of cancer survivorship - definitions. Available at: http://cancercontrol.cancer.gov/ocs/statistics/definitions. html. Accessed September 9, 2018.

3. Deimling GT, Bowman KF, Wagner LJ. Cancer survivorship and identity among long-term survivors. Cancer Invest. 2007;25(8):758-765.

4. Meadows AT, Varricchio C, Crosson K, Harlan L, McCormick P, Nealon E, Smith M, et al. Research issues in cancer survivorship: report of a workshop sponsored by the Office of Cancer Survivorship, National Cancer Institute. Cancer Epidemiol Biomarkers Prev. 1998;7(12):11451151.

5. Khan NF, Rose PW, Evans J. Defining cancer survivorship: a more transparent approach is needed. J Cancer Surviv. 2012;6(1):33-36.

6. Cancer Research UK. 2006. Cancer Stats. Survival-UK. Available at: https://www.cancerresearchuk.org/healthprofessional/cancer-statistics-for-the-uk. Accessed September 9, 2018.

7. Ogle KS, Swanson GM, Woods N, Azzouz F. Cancer and comorbidity: redefining chronic diseases. Cancer. 2000;88(3):653-663.

8. Coebergh JW, Janssen-Heijnen ML, Post PN, Razenberg PP. Serious co-morbidity among unselected cancer patients newly diagnosed in the southeastern part of The Netherlands in 1993-1996. J Clin Epidemiol.
1999;52(12):1131-1136.

9. Fu MR, Axelrod D, Guth AA, Cleland CM, Ryan CE, Weaver KR, Qiu JM, et al. Comorbidities and quality of life among breast cancer survivors: a prospective study. J Pers Med. 2015;5(3):229-242.

10. De Marco MF, Janssen-Heijnen ML, van der Heijden LH, Coebergh JW. Comorbidity and colorectal cancer according to subsite and stage: a population-based study. Eur J Cancer. 2000;36(1):95-99.

11. Piccirillo JF, Feinstein AR. Clinical symptoms and comorbidity: significance for the prognostic classification of cancer. Cancer. 1996;77(5):834-842.

12. Koroukian SM, Murray P, Madigan E. Comorbidity, disability, and geriatric syndromes in elderly cancer patients receiving home health care. J Clin Oncol. 2006;24(15):2304-2310.

13. Land LH, Dalton SO, Jensen MB, Ewertz M. Impact of comorbidity on mortality: a cohort study of 62,591 Danish women diagnosed with early breast cancer, 19902008. Breast Cancer Res Treat. 2012;131(3):1013-1020.

14. Jorgensen TL, Hallas J, Friis S, Herrstedt J. Comorbidity in elderly cancer patients in relation to overall and cancerspecific mortality. Br J Cancer. 2012;106(7):1353-1360.

15. Gronberg BH, Sundstrom S, Kaasa S, Bremnes RM, Flotten $\mathrm{O}$, Amundsen T, Hjelde HH, et al. Influence of comorbidity on survival, toxicity and health-related quality of life in patients with advanced non-small-cell lung cancer receiving platinum-doublet chemotherapy. Eur J Cancer. 2010;46(12):2225-2234.

16. Sogaard M, Thomsen RW, Bossen KS, Sorensen HT, Norgaard M. The impact of comorbidity on cancer survival: a review. Clin Epidemiol. 2013;5(Suppl 1):3-29.

17. Gurney J, Sarfati D, Stanley J. The impact of patient comorbidity on cancer stage at diagnosis. Br J Cancer. 2015;113(9):1375-1380.

18. Geraci JM, Escalante CP, Freeman JL, Goodwin JS. Comorbid disease and cancer: the need for more relevant conceptual models in health services research. J Clin Oncol. 2005;23(30):7399-7404.

19. Park SM, Lim MK, Shin SA, Yun YH. Impact of prediagnosis smoking, alcohol, obesity, and insulin resistance on survival in male cancer patients: National Health Insurance Corporation Study. J Clin Oncol. 2006;24(31):50175024.

20. Shin DW, Nam JH, Kwon YC, Park SY, Bae DS, Park CT, $\mathrm{Cho} \mathrm{CH}$, et al. Comorbidity in disease-free survivors of cervical cancer compared with the general female population. Oncology. 2008;74(3-4):207-215.

21. Goytia EJ, Lounsbury DW, McCabe MS, Weiss E, Newcomer M, Nelson DJ, Brennessel D, et al. Establishing a general medical outpatient clinic for cancer survivors in a public city hospital setting. J Gen Intern Med. 2009;24(Suppl 2):S451-455.

22. Braithwaite D, Tammemagi CM, Moore DH, Ozanne EM, Hiatt RA, Belkora J, West DW, et al. Hypertension is an independent predictor of survival disparity between African-American and white breast cancer patients. Int J Cancer. 2009;124(5):1213-1219.

23. Mouhayar E, Salahudeen A. Hypertension in cancer pa- 
tients. Tex Heart Inst J. 2011;38(3):263-265.

24. Choi KH, Park SM, Lee K, Kim KH, Park JS, Han SH. Prevalence, awareness, control, and treatment of hypertension and diabetes in korean cancer survivors: a cross-sectional analysis of the fourth and fifth Korea national health and nutrition examination surveys. Asian Pac J Cancer Prev. 2013;14(12):7685-7692.

25. Weaver KE, Foraker RE, Alfano CM, Rowland JH, Arora NK, Bellizzi KM, Hamilton AS, et al. Cardiovascular risk factors among long-term survivors of breast, prostate, colorectal, and gynecologic cancers: a gap in survivorship care? J Cancer Surviv. 2013;7(2):253-261.

26. Enright KA, Krzyzanowska MK. Control of cardiovascular risk factors among adult cancer survivors: a populationbased survey. Cancer Causes Control. 2010;21(11):18671874.

27. Guy GP, Jr., Yabroff KR, Ekwueme DU, Rim SH, Li R, Richardson LC. Economic burden of chronic conditions among survivors of cancer in the United States. J Clin Oncol. 2017;35(18):2053-2061.

28. Nekhlyudov L, Lacchetti C, Davis NB, Garvey TQ, Goldstein DP, Nunnink JC, Ninfea JIR, et al. Head and neck cancer survivorship care guideline: American society of clinical oncology clinical practice guideline endorsement of the American cancer society guideline. J Clin Oncol. 2017;35(14):1606-1621.

29. Institute of Medicine (US) Committee on Medicare Coverage of Routine Thyroid Screening; Stone MB, Wallace $\mathrm{RB}$, editors. Medicare coverage of routine screening for thyroid dysfunction. Washington (DC): National Academies Press (US); 2003. 3, Prevalence and Consequences of Thyroid Dysfunction. Available from: https://www. ncbi.nlm.nih.gov/books/NBK221535. Accessed September $9,2018$.

30. Chin HB, Jacobson MH, Interrante JD, Mertens AC, Spencer JB, Howards PP. Hypothyroidism after cancer and the ability to meet reproductive goals among a cohort of young adult female cancer survivors. Fertil Steril. 2016;105(1):202-207 e201-202.

31. Keating NL, Norredam M, Landrum MB, Huskamp HA, Meara E. Physical and mental health status of older long-term cancer survivors. JAm Geriatr Soc. 2005;53(12):2145-2152.

32. Stava CJ, Beck ML, Feng L, Lopez A, Busaidy N, Vassilopoulou-Sellin R. Diabetes mellitus among cancer survivors. J Cancer Surviv. 2007;1(2):108-115.

33. Griffiths RI, Danese MD, Gleeson ML, Valderas JM. Epidemiology and outcomes of previously undiagnosed diabetes in older women with breast cancer: an observational cohort study based on SEER-Medicare. BMC Cancer. 2012;12:613.

34. Sanchez Peralta AM, Oliveras-Lopez MJ, Perez Gonzalez R, Martinez Martinez F, Lopez-Garcia de la Serrana $\mathrm{H}$. Prevalence of diabetes in a cancer population in a Malaga hospital. Nutr Hosp. 2012;27(2):456-462.

35. Karlin NJ, Dueck AC, Cook CB. Cancer with diabetes: prevalence, metabolic control, and survival in an academic oncology practice. Endocr Pract. 2012;18(6):898-905.

36. Lega IC, Pole JD, Austin PC, Lau C, Nathan PC, Baxter NN. Diabetes risk in childhood cancer survivors: a population-based study. Can J Diabetes. 2018;42(5):533-539.

37. Armenian SH, Yang D, Teh JB, Atencio LC, Gonzales A, Wong FL, Leisenring WM, et al. Prediction of cardiovascular disease among hematopoietic cell transplantation survivors. Blood Adv. 2018;2(14):1756-1764.

38. Adams MJ, Lipshultz SE. Pathophysiology of anthracycline- and radiation-associated cardiomyopathies: implications for screening and prevention. Pediatr Blood Cancer. 2005;44(7):600-606.

39. Heidenreich PA, Hancock SL, Lee BK, Mariscal CS, Schnittger I. Asymptomatic cardiac disease following mediastinal irradiation. J Am Coll Cardiol. 2003;42(4):743749.

40. Hooning MJ, Botma A, Aleman BM, Baaijens MH, Bartelink H, Klijn JG, Taylor CW, et al. Long-term risk of cardiovascular disease in 10-year survivors of breast cancer. J Natl Cancer Inst. 2007;99(5):365-375.

41. Smith LA, Cornelius VR, Plummer CJ, Levitt G, Verrill M, Canney P, Jones A. Cardiotoxicity of anthracycline agents for the treatment of cancer: systematic review and meta-analysis of randomised controlled trials. BMC Cancer. 2010;10:337.

42. Ewer SM, Ewer MS. Cardiotoxicity profile of trastuzumab. Drug Saf. 2008;31(6):459-467.

43. Chen HX, Cleck JN. Adverse effects of anticancer agents that target the VEGF pathway. Nat Rev Clin Oncol. 2009;6(8):465-477.

44. Tsai HT, Marshall JL, Weiss SR, Huang CY, Warren JL, Freedman AN, Fu AZ, et al. Bevacizumab use and risk of cardiovascular adverse events among elderly patients with colorectal cancer receiving chemotherapy: a population-based study. Ann Oncol. 2013;24(6):1574-1579.

45. Hampton T. Cancer therapy can be hard on the heart: researchers aim to explain - and avoid - cardiotoxicity. JAMA. 2010;303(11):1019-1020.

46. Mertens AC, Liu Q, Neglia JP, Wasilewski K, Leisenring W, Armstrong GT, Robison LL, et al. Cause-specific late mortality among 5-year survivors of childhood cancer: the Childhood Cancer Survivor Study. J Natl Cancer Inst. 2008;100(19):1368-1379.

47. Miller KD, Siegel RL, Lin CC, Mariotto AB, Kramer JL, Rowland JH, Stein KD, et al. Cancer treatment and survivorship statistics, 2016. CA Cancer J Clin. 2016;66(4):271-289.

48. American Cancer Society. 2017. Breast cancer survival rates. Available at: https://www.cancer.org/cancer/breastcancer/understanding-a-breast-cancer-diagnosis/breastcancer-survival-rates.html. Accessed September 9, 2018.

49. Cook MB, McGlynn KA, Devesa SS, Freedman ND, Anderson WF. Sex disparities in cancer mortality and survival. Cancer Epidemiol Biomarkers Prev. 2011;20(8):16291637. 